\title{
Die maskierte Gesellschaft
}

\section{Staunen}

Wer in diesen Tagen mit offenen Augen durch die Straßen geht, kommt aus dem Staunen nicht mehr heraus. Viele Menschen, jung und alt, tragen Masken aller Art und aller Farben. Die Maskenvielfalt kennt keine Grenzen. Jeder trägt die Maske, wie er meint: Manche bedecken nahezu das ganze Gesicht, andere nur den Mund, manche liegen dicht an, andere stehen weit ab. Jede Art medizinischer Masken wird getragen, chirurgischer Mund-Nasen-Schutz, FFP-Masken, ohne und mit Ventil. Die meisten tragen Stoffmasken.

Wieder andere haben die Maske vorübergehend abgesetzt, sie liegt am Hals, um jederzeit wieder aufgesetzt werden zu können. Diejenigen, die keine Maske im Freien tragen, haben solche Masken sicher in der Tasche, denn ohne diese kommt man in kein Geschäft und keine Bahn. Also treten sie in ein Geschäft und ziehen die Maske an und fühlen sich sicher. Treten sie aus dem Geschäft, wird die Maske abgenommen und wieder in die Tasche gesteckt. Beim nächsten Geschäft wiederholt sich das Vorgehen. Im Auto darf die Maske nicht getragen werden, also kommt sie auf den Sitz, ins Handschuhfach oder wird am Rückspiegel aufgehängt.

Staunt sonst noch jemand? Offenbar nicht. Denn nichts steht zu lesen, nichts wird berichtet darüber, niemand spricht aus, dass wir alle einen großen Unfug vollziehen, der weder wissenschaftlich begründet, noch plausibel, noch harmlos ist. Alle stimmen täglich zu. Wer nicht folgt, bekommt die überwältigende Überzeugung der Folgsamen zu spüren, denn sie sind sicher, das Richtige zu tun. In einem kürzlichen Editorial des Deutschen Ärzteblatts figuriert das öffentliche Maskentragen zu einem Akt der „Rücksichtsnahme zum Schutz Dritter“ [1].

\section{Fehlende wissenschaftliche Begründung}

Nicht einmal das RKI behauptet, den öffentlichen Maskengebrauch wissenschaftlich begründen zu können. Es behauptet lediglich, dass das situationsbedingte Tragen von Masken ein weiterer Baustein sein kann, um Übertragungen zu verhindern [2]. Hierbei handelt es sich um ein Plausibilitätsargument; weil Masken die Freisetzung von Viren vermindern können, erscheint es sinnvoll, auch diesen Effekt zu nutzen. WHO, ECDC und CDC haben sich ebenfalls für das situationsbedingte Tragen von Masken ausgesprochen, auch diese, ohne eine wissenschaftliche Begründung zu beanspruchen [3-5]. Die letzte Cochrane-Metaanalyse aus jüngster Zeit belegt, dass es eine solche nicht gibt [6].

Die rezente Stellungnahme der DGP kommt demgegenüber zu dem Schluss: „Nicht medizinische Mund-Nasenmasken bieten einen nachgewiesenen Fremdschutz. Ein Selbstschutz ist nicht nachgewiesen, aber wahrscheinlich “ [7]. Dieser ist erstaunlich, da die zitierte Literatur dazu den Fremd- noch den Eigenschutz schwerlich belegen kann. Die dargestellten Filterleistungen verschiedener Masken und Stoffe belegen noch lange keine Effektivität im Setting der Öffentlichkeit bzw. der aktuellen epidemiologischen Situation. Die spärlichen, noch dazu methodisch schwachen und im Ergebnis widersprüchlichen Interventionsstudien beziehen sich auf Haushalte, nicht auf die Öffentlichkeit, Geschäfte oder öffentliche Verkehrsmittel. Dasselbe gilt für eine jüngst publizierte, von der WHO gesponsorte Metaanalyse, die insinuiert, eine Evidenz für das öffentliche Maskentragen gefunden zu haben, die aber ganz überwiegend Studien aus dem Setting des Gesundheitswesens eingeschlossen hat: Von 44 Studien behandelt eine einzige kleinere Fall-Kontroll-Studie das öffentliche Maskentragen [8]. Das Interesse, öffentliches Maskentragen zu unterstützen, verleitet sogar manche prominente Autoren dazu, eigene Beiträge, die eher Kritisches zusammengetragen haben („Masks are not only tools, they are also talismans“ [9], nachträglich in Richtung der uneingeschränkten Befürwortung des öffentlichen Maskentragens zu revidieren [10]. Dies allerdings wirft eher ein fragwürdiges Licht auf ihre Position.

Die situationsabhängige Maskenpflicht wurde von vielen dahingehend verstanden, generell Masken im Freien zu tragen. Gegen dieses Missverständnis erfolgten keine Richtigstellungen von Seiten des RKI bzw. der Politik; vielmehr muss davon ausgegangen werden, dass dieses Verständnis gebilligt wird.

\section{Fehlende Plausibilität}

Fehlende Evidenz für eine Intervention bedeutet natürlich nicht, dass eine Intervention nicht plausibel sein kann. Tatsächlich halten medizinische Masken (sowohl Mund-Nasen-Schutz als auch FFPMasken) Viren in der Ausatemluft ab. Im Setting des Krankenhauses sind medizinische Masken effektiv, weil es sich um geprüfte, standardisierte Produkte handelt, die in definierten Situationen Einsatz finden sollen; die Einhaltung der Regeln des Umgangs mit Masken ist definiert, und diese können auch eingehalten werden. Die Rationale für ein öffentliches, situationsgebundenes Maskentragen besteht darin, dass durch dieses Übertragungen durch asymptomatische Träger der Virusinfektion verhindert werden können.

Die Plausibilität für diese bricht allerdings zusammen, wenn die eingangs geschilderte Realität des Maskentragens in den Blick genommen wird. Dazu sollten folgende Punkte in Erinnerung gerufen werden:

- Masken sind im Setting des Gesundheitswesens nur effektiv, wenn sie adäquat verwendet werden, d.h. die angemessene Maske gewählt und der Sitz auf Dichtigkeit geprüft wird. Stoffmasken werden ausdrücklich nicht empfohlen.

- Masken können kontraproduktiv sein, wenn ihre Verwendung nicht hygienisch korrekt erfolgt; dies schließt die 
Händedesinfektion vor dem Aufsetzen, die Vermeidung einer Berührung der Außenfläche, die Händedesinfektion vor und nach dem Absetzen sowie die Vermeidung der Berührung von Innen- und Außenflächen voraus.

- Durchfeuchtete Masken sollen umgehend ausgetauscht werden.

- Eine Wiederverwendung von Masken ist ausgeschlossen bzw. nur unter definierten Bedingungen ausnahmsweise erlaubt.

Alle diese Regeln, auf die das RKI zu Recht immer wieder hinweist und die im Setting des Gesundheitswesens schon schwer genug umzusetzen sind, können im öffentlichen Leben nicht eingehalten werden. Es gibt keine Kontrolle über die Qualität der Maske, und es gibt kein verbreitetes Wissen über den hygienisch richtigen Umgang mit der Maske. Es gibt auch keine Möglichkeit, über die Einhaltung irgendwelcher diesbezüglicher Regeln zu wachen. Einfacher gesagt: Es ist schlicht unmöglich, diese Regeln in der Öffentlichkeit einzuhalten bzw. durchzusetzen.

Die Voraussetzungen des hygyienisch korrekten Gebrauchs für die Empfehlung des öffentlichen Maskentragens, von allen Behörden, auch dem RKI [11], ausführlich geschildert, fallen in der Realität stillschweigend unter den Tisch; dass sich die Empfehlung (und die darauf basierende politische Maßgabe) damit eigentlich selbst widerruft, kommt nicht mehr zu Bewusstsein.

Die Filterleistung nichtmedizinischer Masken, die ganz überwiegend in der Öffentlichkeit in Form von Stoffmasken getragen werden, variiert ganz erheblich. Zusammen mit der mehr oder weniger beliebigen Gebrauchsweise der Masken ist eine Abschätzung der tatsächlichen Fremd- oder Eigenschutzwirkung nicht realistisch möglich. Auch und gerade mögliche prospektive Studien zum öffentlichen Maskengebrauch können dieses Problem kaum beheben, da in einer solchen Studie eine Standardisierung der Masken und des Maskengebrauchs erforderlich ist, die ihrerseits eine externe Validität (d.h. die Übertragbarkeit auf die Situation des ungeordne- ten Maskengebrauch im Alltag) konterkariert.

Ein wesentliches Motiv für die Befürwortung des Maskentragens im öffentlichen Raum scheint die Einschätzung der besonderen Gefahr zu sein, die von der Corona-Epidemie ausgeht; dies wird zuweilen auch ausdrücklich so ausgesprochen [12]. Diese Einschätzung scheint jedoch einerseits im Vergleich zur Exzess-Letalität der epidemischen Influenza fraglich, andererseits angesichts der dürftigen Datenlage über die tatsächliche Übersterblichkeit durch die SARS-CoV-2-Infektion ein wenig tragfähiges Argument.

Zuletzt wurde neben einer Übertragung durch Tröpfchen und Oberflächen auch die Übertragung durch Tröpfchenkerne (Aerosole) diskutiert [13]. Hierbei handelt es sich um einen möglichen, aber seltenen Übertragungsweg. Dieser spielt für die Übertragung im Freien keine Rolle; in geschlossenen Räumen wäre eine gute Lüftung die erste präventive Maßnahme. Im Krankenhaus sind medizinische Masken wahrscheinlich protektiv; gerade zum Schutz vor Tröpfchenkernen sind jedoch andere als medizinische Masken nicht geeignet.

\section{Risiken des öffentlichen Maskentragens}

Statt einer Plausibilität stehen wir an dieser Stelle vor zusätzlichen Risiken. Sie bestehen darin, dass eine kontaminierte Maske Übertragungen sogar befördern kann. Sie bestehen auch darin, dass durch das Maskentragen eine falsche Sicherheit suggeriert wird, die die Einhaltung der wichtigsten Maßnahme, des Face-to-Face-Abstands, gefährden kann. Es wird mindestens gesagt werden müssen, dass die marginalen protektiven Effekte des öffentlichen situationsabhängigen Maskentragens durch die offensichtlichen Risiken ausgeglichen werden.

Ein Einwand könnte dann immer noch lauten, dass das Maskentragen als eine Art permanentes Signal des Ausnahmezustands wirksam sein kann, um die Aufmerksamkeit für die Einhaltung der Hygieneregeln aufrecht zu erhalten. Es würde einer Gesellschaft, die sich als Aus- druck einer rationalen Zivilisation versteht, nicht besonders zur Ehre gereichen, wenn es tatsächlich eines solchen Masken-Mummenschanzes bedürfte, um vernünftiges Verhalten zu befördern. Es sollte auch nicht unterschätzt werden, dass die Aufrechterhaltung einer permanenten Alarmstimmung einen Angstpegel unterhalten kann, der für manche Menschen bedrückend, wenn nicht psychisch bedrohlich werden kann; allein das (nicht empfohlene) vielfache Tragen der Masken im Freien ohne Kontakt zu anderen zeigt, wie stark die Wirkung der Alarmstimmung sein kann. Dessen ungeachtet zeichnet sich an dieser Stelle ein viel tiefgründigeres Problem ab. Es stellt sich nämlich die Frage, ob das Maskentragen in der Öffentlichkeit von nun an ein neuer Standard im Umgang mit dem Risiko von Virusinfektionen und damit in unserer Alltagskommunikation werden soll.

\section{Öffentliches Maskentragen als neuer Standard in Zeiten erhöhter Infektionsgefahr?}

Bei der Beurteilung der Plausibilität von Masken in der Öffentlichkeit ist allerdings weitgehend in Vergessenheit geraten, dass für eine Übertragung ein Face-to-Face-Kontakt über eine gewisse Zeitspanne (laut RKI mindestens 15 Minuten) erforderlich ist; d.h. vorübergehende oder auch längere engere Kontakte ohne Gesichtskontakt sind nicht oder kaum relevant. Abstand, Vermeidung eines Gesichtskontakts und Begrenzung der Kontaktzeit sind somit für die Vermeidung einer Übertragung (durch Tröpfchen) ausreichend. Die unwahrscheinliche Möglichkeit, dass es dennoch zu einer Übertragung kommt, kann kaum eine so weitreichende Maßnahme wie die situationsabhängige Maskenpflicht in der Öffentlichkeit begründen.

Aktuell ist unklar, wann die situationsbezogene Maskenpflicht in der Öffentlichkeit eigentlich beendet werden soll. Ohne eine Impfung wird uns das SARSCoV-2, mutmaßlich mit saisonbedingten Schwankungen, bis auf Weiteres begleiten; ob eine Impfung das Coronavirus eradizieren kann, ist ebenfalls keines- 
wegs sicher. Wie anders jedoch kann man den Rückzug von der Maskenpflicht begründen?

Doch auch über das SARS-CoV-2 hinaus stellt sich schon jetzt die Frage, wie denn in den kommenden Herbst-Winter-Zeiten mit der Influenza umgegangen werden soll, die je nach Virusmutanten ein mindestens gleichrangiges, wenn nicht deutlich höheres Risiko darstellt. Die Influenza ist hinsichtlich der Übertragung hauptsächlich über Tröpfchen, z. T. durch Tröpfchenkerne, der Möglichkeit asymptomatischer Infektionen sowie des Phanömens der möglichen „Superspreader“ ganz ähnlich dem SARS-CoV-2 einzuschätzen. Wie soll begründet werden, in einer Influenzasaison auf das situationsbezogene Maskentragen zu verzichten? Dieselbe Frage stellt sich für eine Reihe anderer Viren, wie z.B. Parainfluenzaviren, RS-Viren, Adenoviren, Metapneumoviren, die Atemwegsinfektionen auslösen können. Konsequent zu Ende gedacht, kann hinter jeder mutmaßlichen Erkältung ein tödliches Virus stehen; soll demnach in Zukunft jeder Mensch mit Schnupfen öffentlich eine Maske tragen oder in jeder Saison mit gehäuften Atemwegsinfektionen eine generelle öffentliche Maskenpflicht die Konsequenz sein?

Einige Stimmen weisen schon auf den Fernen Osten hin, wo das Maskentragen in den Wintermonaten vielfach selbstverständlich geworden sei. Abgesehen davon, dass diese Stimmen die dortige extreme Luftverschmutzung und die unvergleichlich höhere Bevölkerungsdichte als zusätzlichen maßgeblichen Grund für das öffentliche Maskentragen verkennen, erhebt sich doch nachdrücklich die Frage, ob wir angesichts der fehlenden Evidenz und Plausibilität für eine solch eingreifende Maßnahme unseren kulturellen Standard der offenen Begegnung untereinander, ein unaufgebbares Gut in einer freien Gesellschaft, einfach aufgeben wollen.

Masken verdecken das Gesicht bis auf die Augen. Die Kommunikation mit dem Gegenüber wird maßgeblich eingeschränkt. Eine offene Begegnung ist buchstäblich nicht möglich. Masken können im Gegenteil dazu dienen, sich zu verbergen, sich unkenntlich zu machen; die Assoziation beim Anblick von Personen, die speziell schwarze Stoffmasken tragen, mit Überfällen kommt nicht von ungefähr. Gesetzlich gilt in Deutschland ein Vermummungsverbot, nicht ohne Grund. In der Diskussion um das Tragen einer Burka war das Hauptargument für ein Verbot, dass eine solche die Würde der Frau als selbstständiger Person beeinträchtige. All dies scheint angesichts des neuen Risikos der SARS-CoV-2-Epidemie vergessen.

Das Tragen von Masken über einen längeren Zeitraum ist unangenehm. Zusammen mit der eingeschränkten, man muss sagen: versehrten Kommunikation kann dies zu einer dauerhaft veränderten Stimmungslage führen, die wesentliche Verhaltensmuster verändert. Das öffentliche Maskentragen, demnächst womöglich regelmäßig über mehrere Monate, wird den öffentlichen Raum nachhaltig beschädigen. Es wird das Zusammenkommen in Restaurants und Hotels, in öffentlichen Räumen beeinträchtigen und das Kaufverhalten dahingehend verändern, dass noch mehr online eingekauft wird. Dies aber kann Folgen bis hin zum Zusammenbruch des städtischen Raums, wie wir ihn jetzt kennen, bedeuten, einhergehend mit einer beispiellosen Pleitewelle des Einzelhandels und der Gastronomie.

Auch wenn das generelle Maskentragen (also auch im Freien) mit Sicherheit sinnlos und kontraproduktiv ist, bleibt zu fragen, ob das situationsbedingte Maskentragen in bestimmten Situation dennoch sinnvoll sein kann. Im Freien ist das Übertragungsrisiko am geringsten; Großveranstaltungen können aufgrund der längeren engen Kontakte dennoch ein Risiko darstellen. Öffentliche geschlossene Räumlichkeiten (z. B. Geschäfte, öffentlichen Verkehrsmittel, Museen, Kirchen) stellen sicher das größere Risiko dar. Ein Maskentragen in diesen Risikosituationen kann eine Maßnahme sein, wenn zwei Bedingungen erfüllt sind:

1. eine Situation hoher Inzidenz

2. eine Abgabe von standardisierten Masken mit schriftlicher und/oder mündlicher Instruktion, wie diese zu handhaben sind

3. eine vordefinierte zeitliche Begrenzung dieser Maßgabe in Abhängigkeit von Zielgrößen der Eindämmung der Epidemie

Zur Zeit ist aber weder eine solche epidemiologische Situation gegeben noch ein solches standardisiertes Maskentragen vorstellbar. In einer Pandemie-Situation bleiben gerade medizinische Schutzmaterialien immer ein begrenztes Gut. Bevor in solch einer Situation der Schluss gezogen wird, dass irgendein Schutz besser ist als gar keiner und dafür jeder Preis recht erscheint, sollte in Erinnerung gerufen werden, dass es nicht Aufgabe der Medizin sein kann, Risiken um jeden Preis zu beseitigen; vielmehr kann es nur darum gehen, Risiken im Rahmen einer verantwortungsbewussten Güterabwägung um einen maßvollen Preis zu reduzieren. Im Übrigen bleibt es dann bei der Verantwortung jedes möglichst gut informierten Einzelnen, welchen Rest-Risiken dieser sich aussetzt.

In dem bereits zitierten Editorial des „Deutschen Ärzteblatts“ heißt es, dass über die Entwicklung eines Impfstoffs hinaus „... neue Antworten auf das, was ,Normalität' für uns künftig ausmacht“, erforderlich seien, und dazu gehört nach Meinung des Autors die „... in Zeiten erhöhter Infektionsgefahr selbstverständliche Nutzung von Schutzmasken in der Öffentlichkeit“ [1]. Sätze wie diese drücken ein verändertes Bewusstsein aus, das nicht mehr reflektiert, auf welcher Grundlage einmal Entscheidungen wie das situationsabhängige öffentliche Maskentragen gefallen sind. Sie zeugen andererseits von einem fehlenden Problembewusstsein für die Folgen der „neuen Antworten“.

Tatsächlich sind wir gezwungen, „neue Antworten auf das, was ,Normalität' für uns künftig ausmacht", zu finden. Die meisten wichtigen Fragen zur Bewertung der SARS-CoV-2-Epidemie und dem Umgang mit ihr sind noch offen und können erst mit einem gewissen Abstand zuverlässig beantwortet werden. Es ist aber schon jetzt deutlich, dass eine wesentliche Frage, die sich aus der Corona-Epidemie ergibt, die nach unserem Verhältnis zu einem Risiko darstellt. Zu dieser Frage gehören Aspekte wie „Wie wird ein Risiko bewertet?“ ebenso wie „Was kostet es, 
ein Risiko zu minimieren?“ und „Welches Risiko und welche Kosten sind wir bereit auf uns zu nehmen?". Wenn es um Kosten geht, in diesem Fall um gesundheitliche und kulturelle Kosten, dann muss die Beweislast für die Notwendigkeit und Effektivität einer Intervention auf Seiten der Intervention liegen.

Unsere aktuell maskierte Gesellschaft meint, sich vor einem Risiko zu schützen, dabei versucht sie nur, sich vor ihrer Angst zu schützen, und scheint dafür bereit, Kosten auf sich zu nehmen, die sie in ihrer Tragweite nicht ermessen kann. Angesichts dessen ist es dringend geboten, zu einem neuen Umgang mit Risiken sowie konkret zu einer neuen Risikobeurteilung zu kommen. Die Konzentration auf eine Surveillance und die Erkennung und Beendigung von lokalen Ausbrüchen weist durchaus in die richtige Richtung. Die aktuelle Konsequenz kann jetzt nur die Aufhebung der situationsabhängigen öffentlichen Maskenpflicht sein.

Interessenkonflikt

Der Autorinnen/Autoren geben an, dass kein Interessenkonflikt besteht.

\section{Autorinnen/Autoren}

S. Ewig ${ }^{1}$, S. Gatermann ${ }^{2}$, S. Lemmen ${ }^{3}$

1 Thoraxzentrum Ruhrgebiet, Kliniken für Pneumologie und Infektiologie, EVK Herne und Augusta-Kranken-Anstalt Bochum

2 Ruhr-Universität Bochum, Institut für Mikrobiologie (IML), Bochum

3 Universitätsklinikum Aachen, Zentralbereich für Krankenhaushygiene und Infektiologie, Universitätsklinikum Aachen

\section{Korrespondenzadresse}

Prof. Dr. med. Santiago Ewig Thoraxzentrum Ruhrgebiet Kliniken für Pneumologie und Infektiologie, EVK Herne und AugustaKranken-Anstalt Bochum Bergstraße 26

44791 Bochum

E-Mail: ewig@augusta-bochum.de

\section{Literatur}

[1] Maibach-Nagel E. Lockerungen nach der Kontaktsperre: Kein Zurück zur alten Normalität. Dtsch Arztebl 2020; 117: A-1089/ B-917

[2] RKI. https://www.rki.de/DE/Content/Infekt/EpidBull/Archiv/2020/Ausgaben/ 19_20.pdf?_blob=publicationFile

[3] WHO. https://www.who.int/influenza/publications/public_health_measures/publication/en/

[4] ECDC. https://www.ecdc.europa.eu/sites/ default/files/documents/COVID-19-use-face-masks-community.pdf

[5] CDC. https://www.cdc.gov/coronavirus/ 2019-ncov/prevent-getting-sick/cloth-face-cover.html

[6] Jefferson T, Jones M, Al Ansari AL et al. Physical interventions to interrupt or reduce the spread of respiratory viruses. Part 1 - Face masks, eye protection and person distancing: systematic review and metaanalysis. https://www.medrxiv.org/content/10.1101/2020.03.30.20047217v2

[7] Dellweg D, Lepper PM, Nowak D et al. Stellungnahme der DGP zur Auswirkung von Mund-Nasenmaskenauf den Eigen- und Fremdschutz bei aerogen übertragbaren Infektionen in der Bevölkerung. https:// pneumologie.de/fileadmin/user_upload/ COVID-19/2020-05-08_DGP_Masken.pdf

[8] Chu DK, Elie A, Duda S et al. Physical distancing, face masks, and eye protection to prevent person-to-person transmission of SARS-CoV-2 and COVID-19: a systematic review and meta-analysis. Lancet 2020. doi:10.1016/S0140-6736(20)31142-9

[9] Klompas M, Morris CA, Sinclair J et al. Universal Masking in Hospitals in the Covid-19 Era. N Engl J Med 2020; 382: e63

[10] Klompas M, Morris CA, Shenoy ES. Universal Masking in the Covid-19 Era. N Engl Med 2020. doi:10.1065/NEJMc2020836

[11] RKI. https://www.bfarm.de/SharedDocs/ Risikoinformationen/Medizinprodukte/DE/ schutzmasken.html

[12] Greenhalgh T, Schmid MB, Czypionka T et al. Face masks for the public during the covid-19 crisis. BMJ 2020; 369: m1435. doi:10.1136/bmj.m1435

[13] Morgenstern J. Aerosols, Droplets, and Airborne Spread. https://first10em.com/aerosols-droplets-and-airborne-spread/

Publikationshinweis

Leserbriefe stellen die Meinung des Autors dar und entsprechen nicht unbedingt der Meinung von Herausgebern oder Verlag. Herausgeber und Verlag behalten sich vor, Leserbriefe nicht, gekürzt oder in Auszügen zu veröffentlichen.

Bibliografie

DOI https://doi.org/10.1055/a-1199-4525

Pneumologie 2020; 74: 405-408

(c) Georg Thieme Verlag KG

Stuttgart · New York

ISSN 0934-8387 